\title{
Post-Rehabilitation Adapted-Yoga at the YMCA for Adults with Acquired Brain Injury: A Feasibility and Pilot Study
}

\author{
Kristine K Miller ${ }^{1 *}$, Richard Burris ${ }^{2}$, Hilary Nuest ${ }^{3}$, Arianne Mason ${ }^{4}$, Arlene A Schmid ${ }^{5}$, Carol Hanna ${ }^{6}$ and \\ Monica Barringer ${ }^{7}$
}

${ }^{1}$ Department of Physical Therapy, Indiana University, USA

${ }^{2}$ Anderson Regional Campus, Saint Vincent Health, USA

${ }^{3}$ Physical Therapist, IU Health, USA

${ }^{4}$ Physical Therapist, GO PT PLLC Seattle, WA

${ }^{5}$ Department of Occupational Therapy, Colorado State University, USA

${ }^{6}$ Yoga Therapist, CYT, YMCA of Madison, USA

${ }^{7}$ University of Evansville, SPT, USA

Submission: March 01, 2019; Published: April 01, 2019

*Corresponding author: Kristine K Miller, Department of Physical Therapy, Indiana University, USA

Abstract

Background \& purpose: Adults with acquired brain injury (ABI) (stroke and traumatic brain injury) experience long-term physical performance deficits for which participation in post-rehabilitation exercise is recommended. Community-based adapted-yoga has potential as an exercise modality to promote post-rehabilitation exercise and physical function improvements. The purpose of this study was to examine the feasibility and benefit of adapted-yoga at the local YMCA for post-rehabilitation adults with ABI.

Methods: Participants were recruited by referral from clinical therapists in outpatient rehabilitation centers to participate in adapted-yoga $2 \mathrm{x}$ /week for 8 weeks at the YMCA. Referral tracking, yoga attendance, safety, and intervention fidelity were assessed for feasibility. Participants completed pre and post-yoga assessments of balance (trunk impairment scale [TIS], berg balance scale [BBS], dynamic gait index [DGI]) and walking (10-meter walk test [10MWT] and six-minute walk test [6MWT]) and 8 weeks of adapted-yoga class.

Results: Thirty-two people were referred to the YMCA adapted-yoga program by clinical therapists with 17 (53\%) of referred persons enrolling in the program. Intervention fidelity was $87 \%$ with the standardized adapted-yoga protocol, and the average number of classes attended was 12.82+3.7. Balance (TIS, p<0.001; BBS, p<0.001; DGI, p<0.001), and walking distance (6MWT, p=0.028) all significantly improved after 8-weeks of yoga. Balance confidence did not improve significantly and walking speed did not change.

Discussion \& Conclusion: Clinician referral to community-based programs may be a feasible mechanism to engage persons in postrehabilitation community-based exercise. Adapted-yoga may beneficially impact balance and walking performance in post-rehabilitation adults with ABI.

Keywords: Traumatic brain injury; Physical rehabilitation; Optimizing patients; Complementary and integrative therapy; Feasibility; Clinical therapists; Yoga intervention; Walking speed

Abbreviations: ABI: Acquired Brain Injury; CIT: Complementary and Integrative Therapy; TIS: Trunk Impairment Scale; BBS: Berg Balance Scale; DGI: Dynamic Gait Index; ABC: Activities-Specific Balance Confidence Scale; 10MWT: 10 Meter walk test; 6MWT: 6 Minute Walk Test

\section{Introduction}

Every year over 2.5 million Americans sustain an acquired brain injury (ABI) which includes traumatic brain injury and stroke [1,2]. People with an ABI experience a variety of functional limitations including some key physical performance deficits. [1-5] Recovery of physical performance deficits, such as

impaired balance and limited walking, is often a focus of post-ABI rehabilitation. Even with a focus on balance and walking recovery, many people with $\mathrm{ABI}$ continue to experience balance and walking deficits after completion of physical rehabilitation [3]. Residual chronic physical deficits can be problematic as people transition from rehabilitation to home, and work to maximize 
long-term functional recovery [4,5]. Specifically, balance has been reported as positively associated with quality of life, and balance self-efficacy has been positively associated with activity and participation in people with a stroke [6]. Additionally, it has been reported that walking in the community is highly valued among the post-stroke population [7] and that capacity for community walking positively correlates with measures of balance and walking speed [8]. Thus, strategies to promote longterm management of these physical deficits are vitally important for patients as they leave physical rehabilitation.

One strategy that has evolved to promote long-term management of chronic deficits after $\mathrm{ABI}$ is structured discharge planning or transition management. In fact, improving transitions to home have been reported as key to; optimizing patients' experience in healthcare, improving the health of patients and caregivers, and reducing healthcare costs [9]. One significant component of transitioning home after rehabilitation is participating in appropriate exercise to promote continued recovery from physical function deficits [10-12]. Adaptedyoga, offered in a community-based setting, as part of the rehabilitation to home transition plan may be promising as a venue to promote exercise participation and ongoing recovery of physical function deficits for people with ABI following their discharge from physical rehabilitation [13,14]. Adapted-yoga, a form of complementary and integrative therapy (CIT), has been reported as a possible intervention to improve several physical performance measures when implemented in a controlled clinical research laboratory setting. Specifically, balance, balance self-efficacy, fear of falling, and walking distance have all been reported to improve after participation in an adaptedyoga program [13-17]. While these data strongly suggest that adapted-yoga offered in a controlled research setting is beneficial for improving balance and walking in people with $\mathrm{ABI}$, there is a critical gap in the evidence related to the feasibility and potential impact of adapted-yoga when offered in a communitybased setting for post-rehabilitation adults.

Therefore, the purpose of this study was to investigate the feasibility and impact of a community-based, post-rehabilitation, adapted-yoga program on balance and walking in adults living in the community after ABI. The specific aims of the study were to investigate the:

a) Feasibility of adapted-yoga for people with $\mathrm{ABI}$ in a community-based setting.

b) Benefits of community-based adapted-yoga on balance and walking outcomes in post-rehabilitation patients with ABI.

\section{Methods}

\section{Design}

This was a pilot implementation study designed to assess the feasibility and benefit of community-based adapted-yoga for improving balance, walking speed, and walking distance in people with $\mathrm{ABI}$.

\section{Participants}

Study participants were recruited through local outpatient rehabilitation clinics by referral from clinical physical therapists and clinical occupational therapists as part of discharge planning during the transition out of physical rehabilitation. Inclusion criteria included
a) 18 years of age or older.
b) diagnosis of traumatic brain injury or stroke.
c) finished with physical rehabilitation.
d) able to sit independently.
e) able to follow instructions in a group setting.
f) willing and able to sign informed consent.
g) has transportation to the YMCA.

\section{Feasibility}

Feasibility was assessed by:

a) documenting all referrals from the clinical therapists.

b) tracking reasons for non-enrollment from referred patients.

c) taking attendance of enrolled participants at adaptedyoga classes.

d) attempting a follow-up with enrolled participants who dropped out before completing the adapted-yoga class and post-yoga assessment.

e) tracking adverse events.

f) assessing intervention fidelity of the adapted - yoga intervention.

All tracked items were documented in a spreadsheet by graduate student research assistants. Intervention fidelity was assessed through video files of the yoga instructor leading each session. The video files were reviewed by undergraduate research assistants and compared to the written standardized protocol. Each item on the written standardized protocol was marked as included or not included in the video by the research assistants.

\section{Assessments}

After signing informed consent, participants completed baseline and post-intervention assessments which included demographics (age gender, race, marital status, and level of education), pathology characteristics (diagnosis, time since ABI incident, and most effected side), and outcome measures of interest (balance, walking speed, and walking distance). Outcome measures included: sitting balance (Trunk Impairment 
Scale [TIS]), standing balance (Berg Balance Scale [BBS], walking balance (Dynamic Gait Index [DGI]), balance confidence (Activities-specific Balance Confidence Scale $[\mathrm{ABC}]$ ), walking speed (10 Meter Walk Test [10MWT]), and walking distance (6 Minute Walk Test [6MWT]).

\section{Outcome measures}

\section{Balance outcome measures}

The TIS was developed to quantify trunk control and sitting balance in people with hemiparesis secondary to stroke [18]. The TIS measures 3 components of sitting balance (static, dynamic, and coordination) producing an overall composite score (023) with higher scores indicating better sitting balance [18]. The BBS is a reliable and valid measure of standing balance in people with ABI $[19,20]$. The BBS has 14 items scored 0-4 with a composite score ranging from 56 [21] A higher score represents better balance and a score $>46$ indicates low fall risk [21]. The DGI is a reliable and valid measure of dynamic walking balance in adults with ABI [22-24]. The DGI has 8 items scored 0-3 with a total score ranging from 0-24 [25]. A higher score represents better walking dynamic balance and a score $>19$ indicates low fall risk. 24 The $A B C$ is a reliable and valid measure of confidence in adults with $\mathrm{ABI}$ [26-29]. The $\mathrm{ABC}$ has 16 items that are selfrated on a scale of $0-100 \%$ confident in ability to perform activities without loss of balance [30]. The responses for the individual items are averaged to determine overall balance confidence ranging from $0-100 \%$ [30]. Higher scores represent better balance confidence and scores $>81.1 \%$ represent a low probability of falling [26].

\section{Walking speed measure}

Comfortable (CWS) and fast walking speed (FWS) were measured with the 10MWT, which is a reliable and valid measure of walking speed in adults with ABI [31-36]. Participants were instructed to walk at their normal walking speed for the CWS test, and they were instructed to walk as fast as they could safely for the FWS test. Participants walked a 10-meter path and were timed in the middle 6 meters to allow a warm-up and slow down. Participants made 2 passes at each speed and the times were averaged to determine the speed and recorded in meters per second $(\mathrm{m} / \mathrm{s})$.

\section{Walking distance measure}

The 6MWT is a reliable and valid measure of walking distance in people with ABI [32,33,35,37-40]. Participants walked back and forth along a 30 -meter walkway making as many passes as possible in 6 minutes. Distance was recorded in meters $(\mathrm{m})$.

\section{Intervention}

The intervention was adapted-yoga class at the YMCA offered 2x/week for 8 weeks. The adapted-yoga protocol was described previously and includes breathing, postures, and relaxation [14]. The intervention progressed over the 8-weeks starting with all seated postures and adding standing and supine postures as the class progressed. Each session was led by a yoga therapist with volunteer class assistants and a physical therapist consultant. The class assistants were volunteers from the community who were trained by the physical therapist about stroke and brain injury and safe participant guarding. The physical therapist was also responsible for determining safe and appropriate sit to stand and floor transfer methods for each participant.

\section{Data analysis}

Data were analyzed with SPSS 23.0. Demographic, pathology characteristic, and feasibility data were analyzed with descriptive statistics and baseline \& post-intervention assessment comparison data were analyzed with paired t-tests, $\mathrm{p}<0.05$ (non-parametric if indicated).

\section{Results}

\section{Feasibility}

Thirty-two people were referred to the adapted-yoga program by clinical occupational and physical therapists with 17 (53\%) enrolling in the program. The reasons for non-enrollment were varied and included; lack of interest in yoga or coming to YMCA, no transportation, no time, doing exercises on their own, and no reason (Table 1). All study activities were completed per the IRB approved protocol at the YMCA with no adverse events. The range of class sessions attended for participants who completed the adapted-yoga class and post-yoga assessment was 5-16 classes, mean $12.82+3.7$. Intervention fidelity was $87 \%$ with the standardized protocol previously established in the research laboratory [14]. The only deviation was initiation of standing postures at week 3 instead of week 2. This was an intentional deviation discussed by the lead yoga instructor and consulting PT. The deviation was made due to the lower functioning status of the adapted-yoga class participants (mean baseline BBS score was $31+13$ ). It was felt delaying standing for 1 week ( 2 sessions) would promote safety in the community setting.

\section{Demographics}

Twelve (70\%) of 17 enrolled subjects completed the adapted-yoga class and post-intervention assessments. Reasons for dropping out included loss of transportation, moving out of the area, schedule conflict, and no reason given (Table 1). There were no statistically significant demographic differences between enrolled participants who finished the program and enrolled participants who dropped out (Table 2). The 12 participants who finished the program were included in the physical performance outcome analyses. The average age of the sample $(\mathrm{N}=12)$ was 52 years (range 29-63). Seven (58\%) participants were male, and 11 (91\%) were Caucasian. Eight (66\%) participants were married, and 5(41\%) were college graduates. Ten $(83 \%)$ participants had a diagnosis of stroke while the other 2 had a diagnosis of traumatic brain injury. The average time since ABI was 37 months (range 10-82), and 8 (66\%) experienced more deficits on the left side. 
Table 1: Participant Flow Chart.

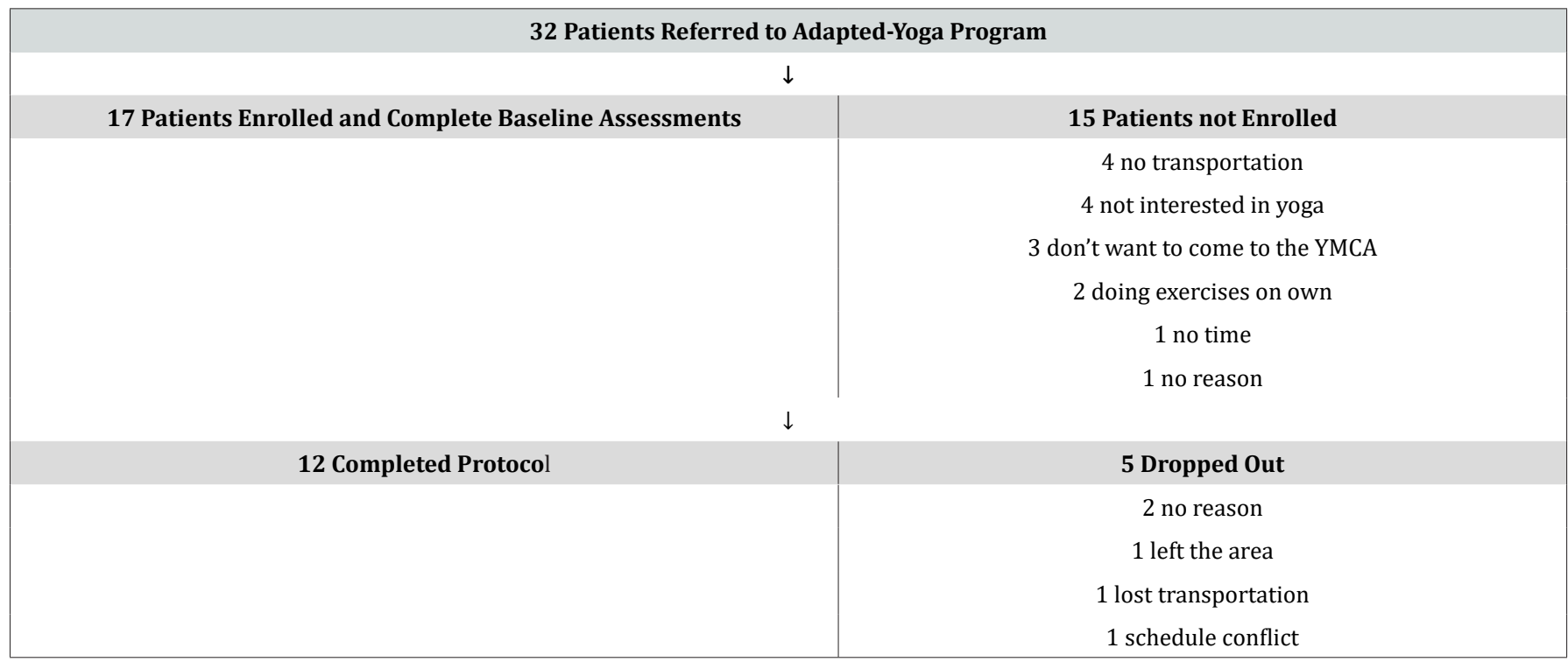

Table 2: Demographic Comparison for Participants (finished vs dropped out).

\begin{tabular}{|c|c|c|c|}
\hline Variables & Finished Program & Dropped Out & N=5 \\
& $\mathbf{N = 1 2}$ & $58 \pm 13$ & 0.36 \\
\hline Age(years) & $52 \pm 11$ & 2 & 49 \\
\hline Gender(male) & 7 & 4 & 0.79 \\
\hline Diagnosis (CVA) & 10 & $41 \pm 50$ & 0.81 \\
\hline Months since DX & $37 \pm 20$ & 3 & 0.35 \\
\hline Side of hemiparesis(left) & 8 & 4 & 0.49 \\
\hline Race (Caucasian) & 11 & 2 & 0.1 \\
\hline Marital status (married) & 8 & 2 & 0.42 \\
\hline Education (college graduate) & 5 & & \\
\hline
\end{tabular}

\section{Balance}

Mean balance scores significantly improved between baseline and post-yoga assessment: BBS $31( \pm 13)$ to $43( \pm 10)$, $\mathrm{p}<0.001$; DGI, $11( \pm 4)$ to $15( \pm 4), \mathrm{p}<0.001$; and TIS (total), 11 $( \pm 4)$ to $18( \pm 3), p<0.001$. Balance confidence $(A B C)$ did not significantly improve (Table 3 ).

Table 3: Paired T-test.

\begin{tabular}{|c|c|c|c|}
\hline Variable & Baseline & Post-Yoga & P-Value \\
\hline TIS (static) & $3 \pm 1$ & $6 \pm 1$ & $<0.001$ \\
\hline TIS (dynamic) & $5 \pm 1$ & $8 \pm 1$ & $<0.001$ \\
\hline TIS (coordination) & $1 \pm 1$ & $3 \pm 1$ & $<0.001$ \\
\hline TIS (total) & $11 \pm 4$ & $18 \pm 3$ & $<0.001$ \\
\hline BBS & $31 \pm 10$ & $43 \pm 10$ & $<0.001$ \\
\hline DGI & $11 \pm 4$ & $15 \pm 4$ & 0.14 \\
\hline ABC (\%) & $56 \pm 27$ & $0.48 \pm 0.35$ & 0.85 \\
\hline 10MWT (CWS) (m/s) & $0.47 \pm 0.33$ & $0.69 \pm 0.52$ & 0.91 \\
\hline 10MWT (FWS) (m/s) & $0.68 \pm 0.56$ & $179 \pm 149$ & 0.028 \\
\hline 6MWT (m) & $147 \pm 124$ & & \\
\hline
\end{tabular}

TIS=trunk impairment scale; BBS-berg balance scale; $D G I=$ dynamic gait index; $A B C=$ activities specific balance confidence scale; 10MWT=10-meter walk test; $\mathrm{CWS}=$ comfortable walking speed; $F W S=$ fast walking speed; $6 \mathrm{MWT}=6$-minute walk test. 


\section{Walking speed \& distance}

Distance walked during the 6MWT significantly improved from 147 meters $( \pm 124)$ to 179 meters $( \pm 149)$, $P=0.028$. Walking speed (10 MWT) did not change (Table 3 ).

\section{Discussion}

The aim of this study was to assess the feasibility and impact of implementing an adapted-yoga protocol in the community for adults with ABI. The results suggest that post-rehabilitation patients can be engaged in community -based exercise through referral relationships between clinics and community-based wellness centers such as the YMCA. In this pilot study, consistent referrals (32 over a 1-year period) to the program by clinical therapists suggests that there may be a need for identified referral sites in the community to facilitate a patient handoff at the time of discharge from rehabilitation. This idea of a patient hand-off between levels of care in the rehabilitation continuum is endorsed by the Transitions of Care Model (TOC) which identifies several key components at the discharge home transition such as medical follow-up, nurse case management, and family education [41-43] but this is the first report to our knowledge that a hand-off to a community wellness program might also be appropriate and important. Clinician referrals to the adapted-yoga program resulted in 53\% of referred patients choosing to enroll. It will be important to address the reasons for non-enrollment moving forward. Additional exercise modalities (non-yoga) will need to be considered for offering in adapted exercise programs at community-based wellness centers to appeal to broader patient preferences. It will also be important to continue efforts to improve effectiveness of the current standard of care (giving a home exercise program (HEP)). Even though adherence rates with HEP are less than ideal [44], some referred patients in this study indicated preference for exercising on their own and not coming to the community-wellness center. Thus, suggesting a multi-modal approach may be needed to engage more patients in regular exercise after discharge from rehabilitation. In addition to suggesting that post-rehabilitation patients can be successfully enrolled in a community-based exercise program, the results of this study also suggest that community-dwelling adults with ABI can improve their balance and walking skills through participation in adapted-yoga at the YMCA.

Balance (sitting, standing, and walking) improved significantly in this sample of people with ABI between baseline and post-yoga assessments. Improved scores on the TIS $(11 \pm 4$ to $18 \pm 3$ ) suggest that adapted-yoga may have a positive impact on trunk control and sitting balance in people with ABI. Trunk control and sitting balance in the post-stroke population have been positively associated with standing balance and walking function [18]. and is an important factor in the recovery of function post-ABI. To our knowledge, this is the first report of the impact of adapted-yoga on sitting balance as measured with the TIS in the ABI population. The improvement in BBS scores
( $31 \pm 13$ to $43 \pm 10$ ) indicates that adapted-yoga may contribute to improved standing balance in the post-ABI population. These results are consistent with results found by Schmid et al. [14] The results in Schmid's project came from a randomized control trial performed in a university-based research laboratory,[14] which is a different setting than the community-based setting in this project. Additionally, the BBS scores in Schmid's study were higher than this project (Schmid, et al: 41.3 to 46.3 , p $<0.001 ; 14$ this study: 31 to $43, \mathrm{p}<0.001$ ). Thus, suggesting that the adaptedyoga protocol may be appropriate and beneficial for people within a wide range of standing balance function levels. It is also important to note that the post-yoga score $(43+10)$ in this sample is still below the low fall risk criterion cut score of 46 , which raises important questions about whether or not progress could continue if participation in adapted-yoga were ongoing and if recruitment through clinical therapist's biases sampling towards patients with lower balance function. Finally, in the area of balance improvements, higher DGI scores $(11 \pm 4$ to $15 \pm 4)$ at post-yoga assessments compared to baseline assessments suggest that walking balance may be positively influenced by participation in adapted-yoga. This is the first report, to our knowledge, of DGI improvements with adapted-yoga after ABI. Overall the potential impact of post-rehabilitation adapted-yoga on balance is an important finding of this study. These findings strongly suggest that participation in an adapted-yoga class in the community after discharge from rehabilitation may encourage ongoing recovery of balance function in patients with $\mathrm{ABI}$. However, in this sample, balance confidence did not improve at a statistically significant level $(\mathrm{P}=0.14)$. This could be due to postyoga balance scores being below the criterion cut scores for low fall risk on the BBS (46) [21] \& DGI (19) [24]. Patients may need to improve even more in balance function before experiencing significant improvement in balance confidence. The insignificant finding with the $\mathrm{ABC}$ could also be related to the small sample size in this pilot study.

Walking distance has been reported as more important to post-stroke patients than walking speed [45]. In this sample of post-rehabilitation patients with $\mathrm{ABI}$, walking distance (meters) improved $(147 \pm 124$ to179 \pm 149$)$ between baseline and postyoga assessments. This outcome is similar to that reported previously by Schmid et al. [16]. Thus, strongly suggesting that participation in a yoga protocol twice a week for a total of 8 weeks can help improve walking distance following ABI independent of setting (clinical research laboratory vs community wellness center). However, consistent with the BBS scores, the 6MWT distances (meters) are lower in this sample than in the sample in Schmid's project (Schmid, et al:288 to 307, $\mathrm{p}<0.001 ; 16$ this study: 147 to $179, \mathrm{p}<0.001$ ), which suggests the positive impact may be present across a wide range of walking distance functional levels. Walking speed did not change in this sample at either comfortable or fast walking speeds.

Overall the results of this study were positive for walking and balance measures. Specifically, the positive results of this 
pilot study suggest that adapted-yoga may be an excellent option for addressing the long-term physical limitations experienced by patients with $\mathrm{ABI}$ after discharge from rehabilitation.

\section{Limitations}

Primary limitations of this study include the relatively small sample size and lack of control group. Future plans include a study with a larger sample size and a control group. Additionally, since the focus of this study was to assess the impact of community-based adapted-yoga on people with $\mathrm{ABI}$, the results cannot be generalized outside of this population.

\section{Conclusion}

This community-based pilot implementation and feasibility trial is an important step in establishing post-rehabilitation exercise options for people with ABI. The patient hand-off from clinic to community wellness center may be an effective mechanism for a smooth transition from rehabilitation to home. A smooth transition from rehabilitation to home is critical for patients to continue their recovery after physical rehabilitation has been finished. Adapted-yoga may be beneficial in promoting ongoing functional recovery of chronic balance and walking distance impairments secondary to ABI after physical rehabilitation has been finished. The results of this study suggest that sitting balance, standing balance, walking balance, and walking distance may all be positively impacted by participation in adapted-yoga. Additionally, the adapted nature of this yoga intervention may make it appropriate for patients at a variety of functional levels.

\section{References}

1. Faul M, Xu L, Wald M, Coronado V (2010) Traumatic brain injury in the United States: Emergency visits, hospitalizations and deaths 20022006. Centers for Disease Control and Prevention, National Center for Injury Prevention and Control.

2. Mozaffarian D, Benjamin E, Go A, et al. (2015) Heart disease and stroke statistics-2015 update: A report from the American Heart Association. Circulation 131(4): e29-322.

3. Desrosiers J, Bourbonnais D, Noreau L, Rochette A, Bravo G, et al (2005) Participation after stroke compared to normal aging. Journal of rehabilitation medicine 37(6): 353-357.

4. Desrosiers J, Noreau L, Rochette A, Bourbonnais D, Bravo G, et al (2006) Predictors of long-term participation after stroke. Disability and Rehabilitation 28(4): 221-230.

5. Michael KM, Allen JK, Macko RF (2005) Reduced ambulatory activity after stroke: The role of balance, gait and cardiovascular fitness. Archives of Physical Medicine and Rehabilitation 86(8): 1552-1556.

6. Schmid AA, Van Puymbroeck M, Altenburger PA, Dierks TA, Miller KK, et al. (2012) Balance and balance self-efficacy are associated with activity and participation after stroke: A cross-sectional study in people with chronic stroke. Archives of Physical Medicine and Rehabilitation 93(6): 1101-1107.

7. Lord S, McPherson K, McNaughton H, Rochester L, Weathrall M (2004) Measurement of community ambulation after stroke: how important and obtainable is it and what measures appear predictive? Archives of Physical Medicine and Rehabilitation 85(2): 234-239.
8. Schmid A, Duncan PW, Studenski S, Lai SM, Richards L, et al. (2007) Improvements in speed-based gait classifications are meaningful. Stroke 38(7): 2096-2100.

9. Berwick DM, Nolan TW, Whittington J (2008) The Triple Aim: Cares Health, And Cost. Health Affairs 27(3): 759-769.

10. Duncan P, Studenski S, Richards L, Gollub S, Lai SM, Reker D, et al. (2003) Randomized clinical trial of therapeutic exercise in subacute stroke. Stroke 34(9): 2173-2180.

11. Leroux A (2005) Exercise training to improve motor performance in chronic stroke: effects of a community-based exercise program. International Journal of Rehabilitation 28(1): 17-23.

12. Pang MY, Eng JJ, Dawson AS, McKay HA, Harris JE (2005) A communitybased fitness and mobility exercsie program for older adults with chronic stroke: A randomized controlled trial. Journal of American Geriatric Society 53(10): 1667-1674.

13. Schmid A, Miller K, Van Puymbroek M, Schalk N (2015) Feasibility abd results of a case study of yoga to improve physical functioning in people with chronic traumatic brain injury. Disability and Rehabilitation 38(9): 914-920.

14. Schmid AA, Van Puymbroeck M, Altenburger PA, Schalk NL, Dierks TA et al. (2012) Post-stroke balance improves with yoga: A randomized pilot study. Stroke 43(9): 2402-2407.

15. Bastille JV, Gill-Body KM (2004) A yoga-based exercise program for people with chronic poststroke hemiparesis. Physical Therapy 84(1): 33-48.

16. Schmid AA, Miller KK, Van Puymbroeck M, DeBaun-Sprague E (2014) Yoga leads to multiple physical improvements after stroke, a pilot study. Complementary Therapies in Medicine 22(6): 994-1000.

17. Schmid AA, Van Puymbroeck M, Koceja D (2010) Effect of 12-week yoga intervention on fear of falling and balance in older adults: A pilot study Archives of Physical Medicine and Rehabilitation 91(4): 576583.

18. Verheyden G, Nieuwboer A, Mertin J, Preger R, Kiekens C, et al. (2004) The Trunk Impairment Scale: a new tool to measure motor impairment of the trunk after stroke. Clinical Rehabilitation 18(3): 326-334.

19. Stevenson TJ (2001) Detecting change in patients with stroke using the Berg Balance Scale. The Australian journal of physiotherapy 47(1): 29-38.

20. Tyson SF, Hanley M, Chillala J, Selley A, Tallis RC (2006) Balance disability after stroke. Physical Therapy 86(1): 30-38.

21. Berg K, Wood-Dauphinee S, Williams J (1995) The Balance Scale: Reliability assessment with elderly residents and patients with an acute stroke. Scand J Rehabil Med 27(1): 27-36.

22. Jonsdottir J, Cattaneo D (2007) Reliability and validity of the dynamic gait index in persons with chronic stroke. Arch Phys Med Rehabil 88(11): 1410-1415.

23. Lin JH, Hsu MJ, Hsu HW, Wu HC (2010) Hsieh CL Psychometric comparisons of 3 functional ambulation measures for patients with stroke. Stroke 41(9): 2021-2025.

24. Medley A (2006) Predicting the probability of falls in community dwelling persons with brain injury: a pilot study. Brain Injury 20(1314): 1403-1408.

25. Wrisley DM, Walker ML, Echternach JL, Strasnick B (2003) Reliability of the dynamic gait index in people with vestibular disorders. Archives of Physical Medicine and Rehabilitation 84(10): 1528-1533.

26. Beninato M, Portney LG, Sullivan PE (2009) Using the International Classification of Functioning, Disability and Health as a framework to 
examine the association between falls and clinical assessment tools in people with stroke. Phys Ther 89(8): 816-825.

27. Botner EM, Miller WC, Eng JJ (2005) Measurement properties of the Activities-specific Balance Confidence Scale among individuals with stroke. Disabil Rehabil 27(4): 156-163.

28. Salbach NM, Mayo NE, Robichaud-Exstrand S, Hanley JA, Richards CL, et al. (2006) Balance and self-efficacy and its relevance to physical function and perceived health status after stroke. Archives of Physical Medicine and Rehabilitation 87(3): 364-370.

29. Inness EL, Howe JA, Niechwiej-Szwedo E, Jaglal SB, Mcllroy WE, et al. (2011) Measuring balance and mobility after after traumatic brain injury: validation of the community balance and mobility scale. Physiotherapy Canada 63(2): 199-208.

30. Powell LE, Myers AM (1995) The activities specific balance confodence scale (ABC). J Gerontol A Biol Sci Med Sci 50A(1): M28-M34.

31. Collen FM, Wade DT, Bradshaw CM (1990) Mobility after stroke: reliability of measures of impairment and disability. International disability studies 12(1): 6-9.

32. Flansbjer UB, Holmback AM, Downham D, Patten C, Lexell J (2005) Reliability of gait performance tests in men and women with hemiparesis after stroke. Journal of rehabilitation medicine 37(2): 7582.

33. Moseley AM, Lanzarone S, Bosman JM, van Loo MA, de Bie RA, et al (2004) Ecological validity of walking speed assessment after traumatic brain injury: a pilot study. The Journal of head trauma rehabilitation 19(4): 341-348.

34. Tyson S, Connell L (2009) The psychometric properties and clinical utility of measures of walking and mobility in neurological conditions: a systematic review. Clin Rehabil 23(11): 1018-1033.

35. Van Loo MA, Moseley AM, Bosman JM, de Bie RA, Hassett L (2004) Test-re-test reliability of walking speed, step length and step width measurement after traumatic brain injury: a pilot study. Brain Inj 18(10): 1041-1048.

36. Wolf SL, Catlin PA, Gage K, Gurucharri K, Robertson R, et al. (1999) Establishing the reliability and validity of measurements of walking time using the Emory Functional Ambulation Profile. Phys Ther 18(10): 1122-1133.

37. Eng JJ, Dawson AS, Chu KS (2004) Submaximal exercise in persons with stroke: Test-retest reliability and concurrent validity with maximal oxygen consumption. Archives of Physical Medicine and Rehabilitation 85(1): 113-118.

38. Fulk GD, Echternach JL (2008) Test-retest reliability and minimal detectable change of gait speed in individuals undergoing rehabilitation after stroke. Journal of Neurological Physical Therapy 32(1): 8-13.

39. Mossberg KA (2003) Reliability of a timed walk test in persons with acquired brain injury. American journal of physical medicine \& rehabilitation 82(5): 385-390.

40. Wevers LE, Kwakkel G, van de Port IG (2011) Is outdoor use of the sixminute walk test with a global positioning system in stroke patients' own neighbourhoods reproducible and valid? Journal of rehabilitation medicine 43(11): 1027-1031.

41. (2008) National Transitions of Care Coalition, MWG, Transition of Care Measures.

42. Broderick JP, Abir (2015) M Transitions of care for stroke patients: Opportunities to improve outcomes. Circulation: Cardiovasc Quality and Outcomes 8(6 Suppl 3): S190-192.

43. Reeves MJ, Hughes AK, Woodward AT, Freddolino PP, Coursaris CK, et al. (2017) Improving transitions in acute stroke patients discharged home:the Michigan stroke transitions trial (MISTT) protocol. BMC Neurology 17(1): 115-1130.

44. Miller K, Porter R, DeBaun-Sprague E, Van Puymbroek M, Schmid A (2016) Exercise after stroke: Patient adherence and beliefs after discharge from rehabilitation. Topics in Stroke Rehabilitation 24(2): 142-148.

45. Combs SA, Van Puymbroeck M, Altenburger PA, Miller KK, Dierks T, et al. (2013) Is wallking faster or walking farther more important to persons with chronic stroke? Disability and Rehabilitation 35(10): 860-867.

Your next submission with Juniper Publishers will reach you the below assets

- Quality Editorial service

- Swift Peer Review

- Reprints availability

- E-prints Service

- Manuscript Podcast for convenient understanding

- Global attainment for your research

- Manuscript accessibility in different formats

( Pdf, E-pub, Full Text, Audio)

- Unceasing customer service

Track the below URL for one-step submission

https://juniperpublishers.com/online-submission.php 